\title{
Survey on Automatic Vehicle Number Plate Localization
}

\author{
B.Balvinder Singh \\ Department of Computer Science and \\ Engineering, V.R.Siddhartha Engineering College, \\ Vijayawada, India.
}

\begin{abstract}
In this paper a survey is being carried out in the field of Automatic license plate localization. Automatic license plate recognition (ALPR) is to extract vehicle license plate information from an image or a sequence of images. The extracted information can be used with or without a database in many applications such as electronic payment systems, freeway and specific road monitoring systems for traffic surveillance. As a real-life application it has to quickly and successfully process license plates under different environmental conditions such as indoors, outdoors, day/night time. The localization or detection of the number plate of the vehicle images is the basis for any ALPR system. This is the first step in any LPR system and is considered as the most crucial step in the ALPR system. The success rate also depends mainly on this stage only. Hence the best localization or detection of the number plate is necessary to get the high recognition rate in any ALPR system. A survey has been made in this field by considering different methods that have been addressed to detect the number plate from the vehicle images. All these methods are categorized based on the features that they have used. At the end, a comparison is made in terms of advantages, drawbacks and possible enhancements.
\end{abstract}

\section{General Terms}

License plate recognition (LPR), Image processing, Automatic Vehicle Number plate localization.

\section{Keywords}

Automatic License Plate Recognition, Edge detection, Number plate localization(NPL), Morphology, Wavelet transform.

\section{INTRODUCTION}

Automatic License Plate Recognition (ALPR) has a wide range of real-life applications such as automatic toll collection, traffic law enforcement, and road traffic monitoring [1]. Reading or locating the license number plate is the main and the first step in determining the identities of parties involved in the traffic incidents. The common aim of these applications is to reduce man power and facilitate to the automatic management. An ALPR system must thus exhibit a high recognition rate and processing speed. For example, drivers normally have little patience when waiting for their vehicle to be recognized by a car parking system. It is also mass surveillance method that uses optical character recognition on images to read vehicle registration plates. They can use existing closed-circuit television or road-rule enforcement cameras, or ones specifically designed for the task. They are used by various police forces and as a method

\author{
V.Hima Deepthi \\ Department of Computer Science and \\ Engineering, V.R.Siddhartha Engineering College, \\ Vijayawada, India.
}

of electronic toll collection on pay-per-use roads and cataloging the movements of traffic or individuals. It can be used to store the images captured by the cameras as well as the text from the license plate, with some configurable to store a photograph of the driver. Systems commonly use infrared lighting to allow the camera to take the picture at any time of the day. This technology tends to be region-specific, owing to plate variation from place to place. An efficient license plate localization system may become the core of fully computerized road traffic monitoring systems. Furthermore, an ALPR system can have two varieties: on-line ALPR system and off-line ALPR system. In an online ALPR system, the localization and interpretation of license plates take place instantaneously from the incoming video frames, enabling real-time tracking of moving vehicles through the surveillance camera. On the other hand, an offline ALPR system captures the vehicle images and stores them in a centralized data server for further processing, i.e. for interpretation of vehicle license plates. ALPRs are cameras mounted on stationary objects [2] (telephone poles, the underside of bridges, etc.) or on patrol cars. The cameras snap a photograph of every license plate that passes them by capturing information on up to thousands of cars per minute.

\section{BASIC CONCEPTS}

Some standards have been given for numbering vehicles. The aspect ratio of the license plate is considered as 1 to 2 for multi line character set and between 3 and 6.5 for single line character set. The following sections describe the specifications of Indian license plates.

\subsection{License Plate Specifications}

The standard specifications of license plates are not same for all countries and may not same even for two countries because every country has their own numbering standards. Here the specifications for indian license plates have mentioned. The numbering format [3] is as follows. AA 11 BB 1111, Where $\mathrm{AA}$ is the state code, 11 is the two digit district code, 1111 is the unique number and $\mathrm{BB}$ are the optional alphabets if the 9999 numbers are used up. An example would be TN $01 \mathrm{CA}$ 3546 , the first two alphabets TN indicate that the vehicle is from the Tamil Nadu state. The next two are representing the district (In this case the capital Chennai). CA 3546 is the unique license plate number.

\subsection{Challenges in ALPR}

There are number of difficulties that the researchers can take as challenges in this field. These include [4]:

1. Poor image resolution, usually because the plate is too far away but sometimes resulting from the use of a low-quality camera.

2. A different font, popular for vanity plates (some countries do not allow such plates, eliminating the problem). This 
creates problem during recognition stage of the number of vehicle.

3. Blurry images, particularly motion blur. The figure below show an example of it.

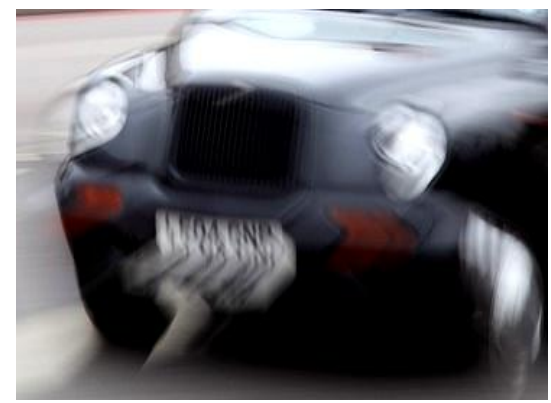

Fig 1: A motion blurred car image

4. Poor lighting and low contrast due to reflection or shadows.

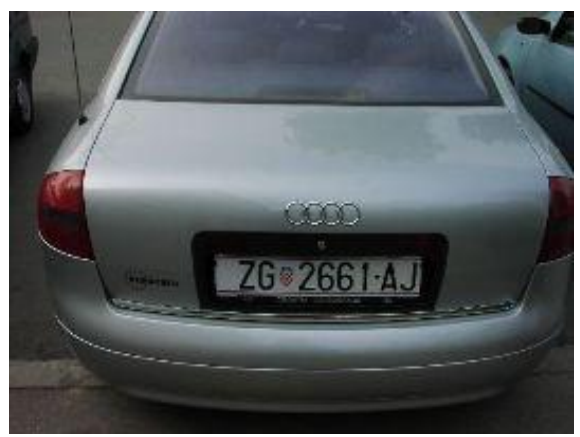

Fig 2: A low contrast vehicle image

5. An object obscuring the plate, quite often a tow bar, or dirt on the plate.

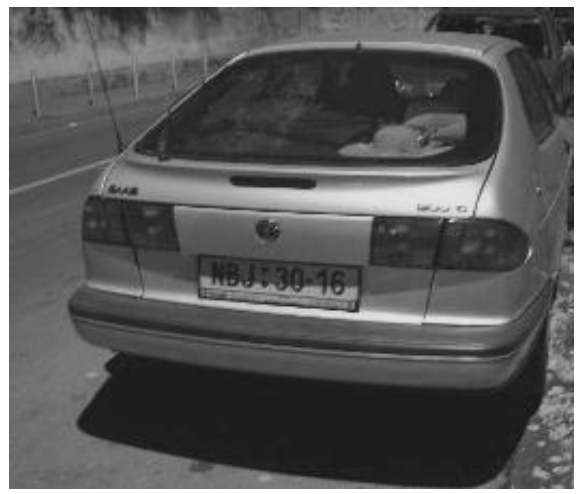

Fig 3: Shadow on the plate region of the car

6. Lack of coordination between countries or states. Two cars from different countries or states can have the same number but different design of the plate.

\section{REVIEW}

Different methods that have been addressed for plate detection of vehicles such as license plate detection [5] using edge information, connected component analysis, color features, texture features, character features etc. This section describes various methods used for localization of the vehicle number plates.

\subsection{Detection of License Plate Using Edge Information}

In this method the license plate is detected using the edge detection techniques. This method detects the license plate characters from the car images taken from various outdoors. Initially the preprocessing is done using median filter. The magnitude of the vertical gradients is used to detect the plate region [6]. The sobel operator is used for edge detection [7] which is computationally inexpensive and reasonably robust to noise. The following figure shows the sobel operator [8].

$$
\left[\begin{array}{ccc}
-1 & 0 & +1 \\
-2 & 0 & +2 \\
-1 & 0 & +1
\end{array}\right]
$$

Fig 4: Sobel operator for vertical edge detection

After detecting edges in the image, the required area is evaluated based on the geometrical features such as the ratio of width and height, size and orientation. The ratio of width to the height is also known as aspect ratio [9]. The following figure 5 shows different stages of detecting license plate using sobel edge detection technique. The standard aspect ratio of the number plates for car images is 1 to 2 for multi line character set and between 3 and 6.5 for single line character set. In [10] Mexican hat operator is used to detect the edges which perform smoothing before extracting edges. This method is simple and straight forward for detecting plate region. Even though it is reasonably robust to noise, cannot produce good results for the images with complex background.

\subsection{Mathematical Morphology for \\ Extraction of Vehicle Number Plates}

To extract vehicle number plates, a methodology based on mathematical morphology is presented. This method has stages such as image enhancement, morphological transformations, morphological gradient, combination of resultant images and extracting the number plate from the objects that are left in the image [11]. As a preprocessing technique here enhancement is used. The technique used for intensity adjustment is histogram equalization. It is the distribution of intensity values equally throughout the image to get more contrast than the original image. Top-hat transformation is applied after enhancement which aims to suppress the dark background and highlights the foreground objects. Top-hat operation is actually the result of subtraction of an opened image from the original one, mathematically:

$$
t h=f-(f o b)
$$

Where, $\mathrm{f}$ is the input image and $\mathrm{b}$ is the structuring element. The selection of structuring element is based on the application and is not fixed. The available structuring 
elements are of shape square, rectangle, diamond and disk. Here the disk shaped structuring element is used. Next to measure the local gray level variation in the image morphological gradient operation is performed. It is the subtraction of an eroded image from its dilated version and can be expressed mathematically:

$$
g=(f \Theta b)-(f o b)
$$

After this step the resulting image changed into binary [12] and all the noise components were removed. Next the plate region is confirmed based on the properties of the plate such as aspect ratio, area of the plate etc. This method gives success rate of $96 \%$ with the images acquired from the highways, toll gates with different conditions.

\subsection{Vehicle License Plate Detection Using Sliding Concentric Windows (SCWs)}

There are three stages used such as for detecting license plate candidate region a novel adaptive segmentation, color verification by using HSI color space, histogram to detect LP region [13]. In the first stage, two concentric windows namely A and B scan the total image from left to right and top to bottom. Then the statistical measurements such as mean and standard deviation in the two windows (boxes) are calculated. If the ratio of the statistical measurements in the two windows exceeds a threshold, the central pixel of the windows is considered to belong to region of interest (ROI). The pixel value in the perspective coordinate $(x, y)$ of the image is set either 0 (not in the region of interest) or 1 (in the region of interest). It is formulated as follows [14]:

$$
I(x, y)= \begin{cases}0, \text { if } & M b / M a \leq T \\ 1, \text { if } & M b / M a>T\end{cases}
$$

Here $\mathrm{M}$ is the statistical measurement and $\mathrm{T}$ is the value of threshold. Next color verification for candidate region. The HSI model separates all the color information, described by hue and saturation, from the intensity component. The color space in the HSI model is not represented by a cube as is done in the RGB color model, because the components are not orthogonal. This is performed using HSI (hue, saturation, intensity) model that verifies green, yellow and white LP respectively. The transformation from RGB to HSI is as follows:

$$
H=\cos ^{-1}\left[\frac{1 / 2[(R-G)+(R-B)]}{\left[(R-G)^{2}+[(R-B)(G-B)]^{1 / 2}\right.}\right]
$$

Finally, candidate LP region is extracted based on the vertical and horizontal position histogram. In [14] after SCWs, morphology is applied instead of using color model. Dilation and erosion are the two morphological operations applied to get the right candidate plate region. These methods can locate more than one plate in an image and the success rate of $96 \%$ is achieved [15]. Since the color is sensitive to lighting conditions fails to detect the LP properly if color information is used.

\subsection{License Plate Localization Using Connected Components Analysis}

An approach for localization of number plates using connected components addressed [16]. First the input image is processed to get quality in order to acquire by the further steps. Color images are changed to the YUV model; Y stands for luma component (the brightness) and $\mathrm{U} V$ are the chromaticness (color) components [17]. Next the image is threshold so that the characters of the number plate were look different from the background. A set of elements which can be a set of license plate characters prepared in the binary image is localized. The thresholded image is then labeled as black and white spots. A spot here is a pixel or group of pixels.

After labeling the spots, the elimination of unwanted spots is done as follows. Spot is eliminated if one of the below given conditions is fulfilled: the width of the spot is smaller than 3 points, the width of the spot is bigger than $1 / 4$ of the input image width, the height of the spot is smaller than 8 points etc. Next the fragments were identified which mostly contains in the candidate region. The neighborhood of each spot in the image is analyzed and if there is another spot of the same size in this neighborhood the spots are grouped. The grouped spots are called a segment. Finally unnecessary spots are removed. This method is straight forward and independent of the plate position but is sensitive to skewed plates.

\subsection{Automated Car License Plate Localization Using Wavelet Analysis}

A method using wavelet analysis for license plate localization for Indian vehicles is provided. The levels in this method are isolation of license plate, horizontal clipping of the plate, vertical clipping of the plate and energy plot. The isolation of LP is accomplished using wavelet transforms. Using two dimensional wavelet transforms [18], a single level decomposition was performed. This produces four components such as approximation (A), diagonal details (D), horizontal details $(\mathrm{H})$, and vertical details $(\mathrm{V})$ respectively. Next horizontal clipping of the plate was performed by using CV1. The following figure 6 shows decomposition of input image into four components using wavelets. Then normalized energy is been plotted. From these the high frequency area shows the typical index along the $y$-axis of the plot. The peak of energy signal is picked by the threshold value. The vertical clipping of the plate was similar to that of horizontal clipping. The horizontal clip is decomposed as approximation (A1), horizontal (H1), vertical (V1) and diagonal (D1). From these V1 gives dominant energy for getting vertical clip. This energy signal shows peak along $\mathrm{x}$-axis with some index range. These clippings are reconstructed by addition and subtraction of the detailed coefficients to get the desired image component. This method robustly locates the license plate from different vehicle images with different lighting conditions. It satisfactorily eliminates all the background unwanted objects in order to get the required region. 


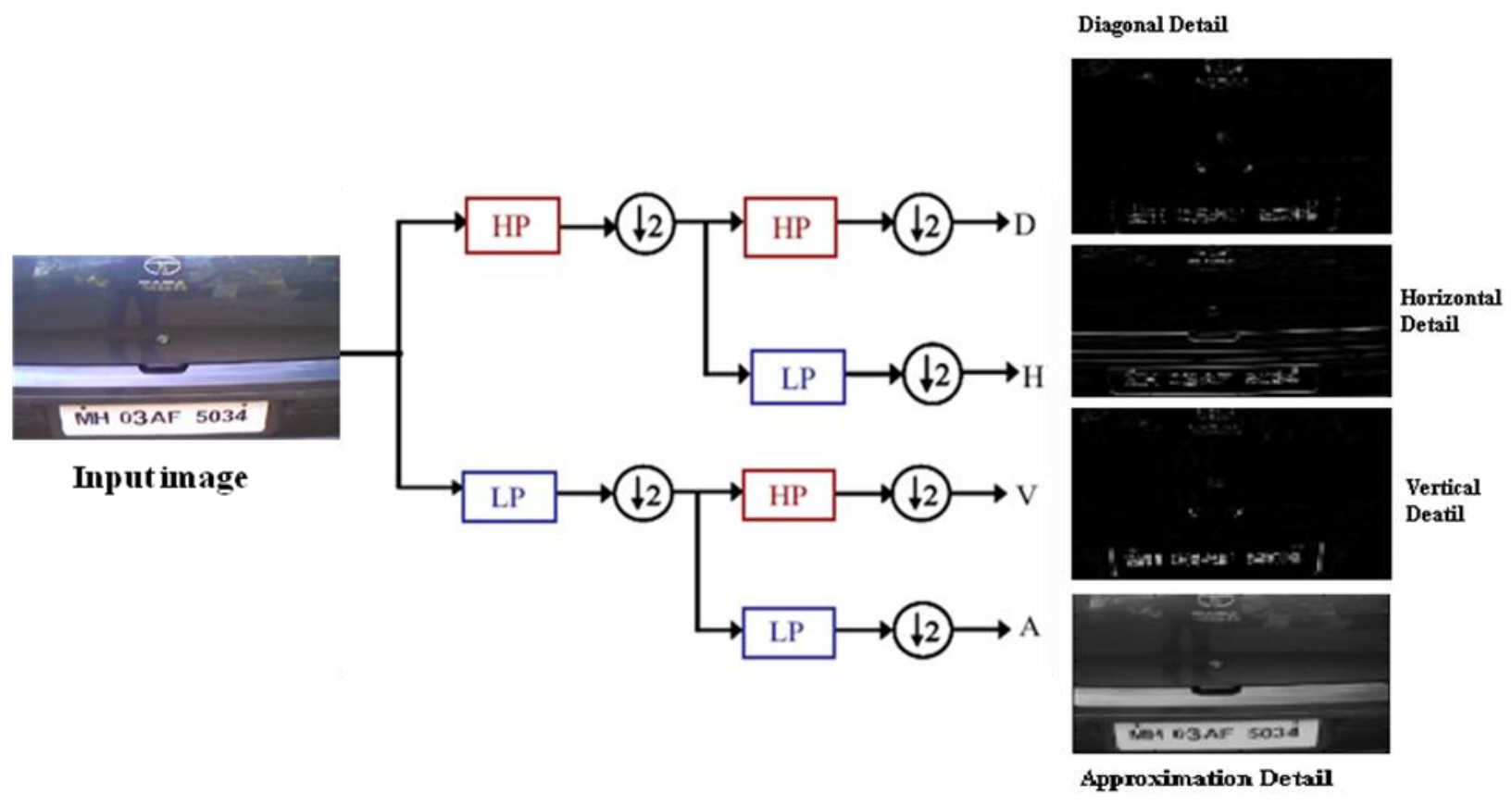

Fig 5: Wavelet decomposition of input car image

\subsection{License Plate Localization Using Color Information}

In this technique the localization or detection of the number plate is performed using color characteristics of the plate [19]. The input is RGB color image. Here only four colors taken into account white, black, red and green. Next color edge detection was done by considering only black-white, redwhite and green-white edge combinations. Now the RGB color space is converted into HSI space. The transform from RGB to HSI is performed using following formulae:

$$
\begin{aligned}
& I=\frac{(r+g+b)}{2} \\
& S=\frac{1-[\min (r, g, b)]}{I} \\
& H=\cos ^{-1}\left\{\frac{(r-g)+(r-b)}{2\left[(r-g)^{2}+(r-b)(g-b)\right]^{1 / 2}}\right\}
\end{aligned}
$$

After that the process of fuzzification was performed as follows. Fuzzy sets provide an elegant tool for uncertainties. The H, S, I and E maps were formulated.

$$
\begin{aligned}
& \mu_{H}(h)=\mu\left(\mu_{r}(h), \mu_{g}(h)\right) \\
& \mu_{S}(h)=\exp (-a s) \\
& \mu_{I}(i)=1-\exp (-a(i-\bar{i})) \\
& \mu_{E}\left(e_{p}\right)=\sum_{k \in N_{p}} e_{k} \exp \left(-a d_{p k}\right)
\end{aligned}
$$

Where, $e_{K}$ is the edge magnitude of the pixel $K$ and $d_{p k}$ is the Euclidean distance from $\mathrm{p}$ to $\mathrm{k}$. Each of these fuzzy maps provides information for locating LP region in the input image. All these maps were combined into a single map $\mathrm{M}$ that provides the detected license plate. This method is less restrictive compared to the previous color based methods and can be easily extended to any color characteristics for different countries. Even though it gives good results but the time complexity of this has to be reduced. Suffers from wrong detection when some parts of the image have same license plate color such as the car body.

\subsection{Number Plate Localization Using Texture Feature}

A method for localization of number plates of Chinese passenger cars was given. The localization was performed based on the number of edge points, the length of license plate area and the number of each line of edge points [20]. The input images were preprocessed by median filter and thereafter by using sobel edge detection the edge points were formed. Binarization of the resultant image was done to reduce the invalid and unwanted portions in the image. The ratio between the number of edge points and the length of license plate area is from 3.9 to 13 . The column range and row range were detected based on the number of edge points, the length of the plate area etc. The approximate localization was done based on the minimum and maximum edge points. As this was not a required region, to get the accurate region aspect ratio taken into consideration. 


\subsection{License Plate Detection Based on Genetic Neural Networks, Morphology and Active Contours}

This method detects license plate by using GNN (genetic neural networks), morphology and contours. The steps in this are preprocessing, processing, training, execution and post processing [21]. Preprocessing was done by using several algorithms such as thresholding, edge filtering and histogram techniques. During processing the preprocessed images were divided into $10 \times 10$ regions. For each region the number of edge points, the amount gray, white and black pixels were calculated. Next in each iteration of training the details of one region can be obtained. The GNN processed these details and give the output for each region that shows whether it belongs to the plate area or not. The images for the training phase were selected in such a way that each image in the set contains a bright plate, sloping plate and a dark one etc. In the execution phase all the activated regions are joined together to get the candidate area. Morphology operations such as opening and closing were applied to remove the unnecessary objects [22] as post processing. The result is a rectangle like region and near to the searched area. Finally, active contour or snake has been used to adopt to the boundary of the license plate.

TABLE 1. Comparison of each of the Localization Methods

\begin{tabular}{|c|c|c|}
\hline Method & Principle & Pros \\
\hline $\begin{array}{c}\text { Using edge } \\
\text { information } \\
{[6][8][9]}\end{array}$ & $\begin{array}{l}\text { The area around } \\
\text { the plate region is } \\
\text { rectangular. }\end{array}$ & $\begin{array}{c}\text { Easy and } \\
\text { straightforward. }\end{array}$ \\
\hline $\begin{array}{c}\text { Using } \\
\text { morphology } \\
{[11][12]}\end{array}$ & $\begin{array}{l}\text { The shape of the } \\
\text { plate. }\end{array}$ & Easy to implement. \\
\hline $\begin{array}{c}\text { Using sliding } \\
\text { concentric } \\
\text { windows [13] } \\
{[14]}\end{array}$ & $\begin{array}{c}\text { Scanning in } \\
\text { vertical and } \\
\text { horizontal } \\
\text { directions to get } \\
\text { edges }\end{array}$ & $\begin{array}{l}\text { Produces good } \\
\text { results with different } \\
\text { illumination } \\
\text { conditions }\end{array}$ \\
\hline $\begin{array}{c}\text { Using } \\
\text { connected } \\
\text { components } \\
{[16]}\end{array}$ & $\begin{array}{c}\text { Finding a } \\
\text { connected object } \\
\text { whose dimension } \\
\text { is like a plate. }\end{array}$ & $\begin{array}{l}\text { Simple, independent } \\
\text { of the position of the } \\
\text { plate. }\end{array}$ \\
\hline $\begin{array}{c}\text { Using } \\
\text { wavelets [18] }\end{array}$ & $\begin{array}{l}\text { Decomposition } \\
\text { and } \\
\text { reconstruction }\end{array}$ & $\begin{array}{l}\text { Successfully } \\
\text { eliminates all the } \\
\text { noise components } \\
\text { and preserves only } \\
\text { the plate region }\end{array}$ \\
\hline $\begin{array}{c}\text { Using color } \\
\text { information } \\
{[19]}\end{array}$ & $\begin{array}{l}\text { Particular color } \\
\text { on the plate }\end{array}$ & $\begin{array}{c}\text { Able to detect } \\
\text { deformed license } \\
\text { plates }\end{array}$ \\
\hline $\begin{array}{l}\text { Using two or } \\
\text { more } \\
\text { features[5] }\end{array}$ & $\begin{array}{l}\text { Combining two o } \\
\text { features more } \\
\text { effective }\end{array}$ & $\begin{array}{l}\text { More reliable and } \\
\text { effective }\end{array}$ \\
\hline
\end{tabular}

\section{COMPARISON OF EACH OF THE LOCALIZATION METHODS}

Here a comparison is made in terms of advantages, disadvantages and the fundamental rule based on which each of the method was developed. The following table 1 is used to illustrate it. The table 1 describes the advantage and the principle based on which each of the license plate localization or detection performed. The following table 2 shows the disadvantage of each of the localization method with possible enhancements.

\section{CONCLUSION \& FUTURE WORK}

In this study various methods for license plate localization of vehicle images is presented. With rapid development of transportation technology, monitoring of vehicles is necessary for various purposes. The main step in any recognition system of this field is localization or detection of number plate of vehicle images hence the overall correctness of the system depends on it. Due to various environmental conditions and independent standards among the countries, localization of the number plate is a challenging problem in the world.

TABLE 2: Cons and possible future enhancements of each of the localization method.

\begin{tabular}{|c|c|c|}
\hline Method & Cons & $\begin{array}{c}\text { Possible Future } \\
\text { Enhancement }\end{array}$ \\
\hline $\begin{array}{c}\text { Using edge } \\
\text { information } \\
{[6][8][9]}\end{array}$ & $\begin{array}{l}\text { Not suitable for } \\
\text { complex images } \\
\text { due to unwanted } \\
\text { edges. }\end{array}$ & $\begin{array}{l}\text { Accurate } \\
\text { Preprocessing } \\
\text { needed. }\end{array}$ \\
\hline $\begin{array}{c}\text { Using } \\
\text { morphology } \\
{[11][12]}\end{array}$ & $\begin{array}{l}\text { May get deformed } \\
\text { boundaries }\end{array}$ & $\begin{array}{l}\text { Incorporating } \\
\text { Adaptive } \\
\text { Thresholding }\end{array}$ \\
\hline $\begin{array}{c}\text { Using sliding } \\
\text { concentric } \\
\text { windows [13] } \\
\text { [14] }\end{array}$ & $\begin{array}{l}\text { Sensitive to angle } \\
\text { of view and } \\
\text { physical } \\
\text { appearance }\end{array}$ & $\begin{array}{c}\text { Skew correction } \\
\text { and High } \\
\text { Dynamic } \\
\text { Range(HDR) } \\
\text { imaging required. }\end{array}$ \\
\hline $\begin{array}{l}\text { Using connected } \\
\text { components [16] }\end{array}$ & $\begin{array}{l}\text { May generate } \\
\text { broken objects }\end{array}$ & $\begin{array}{l}\text { Need one or two } \\
\text { co-operating } \\
\text { methods. }\end{array}$ \\
\hline $\begin{array}{c}\text { Using wavelets } \\
{[18]}\end{array}$ & $\begin{array}{c}\text { Not directly } \\
\text { suitable for skewed } \\
\text { plate }\end{array}$ & $\begin{array}{l}\text { Skew correction } \\
\text { needed. }\end{array}$ \\
\hline $\begin{array}{c}\text { Using color } \\
\text { information [19] }\end{array}$ & $\begin{array}{c}\text { Sensitive to } \\
\text { lighting conditions }\end{array}$ & $\begin{array}{l}\text { Soft computing } \\
\text { techniques } \\
\text { needed. }\end{array}$ \\
\hline $\begin{array}{l}\text { Using texture } \\
\qquad[20]\end{array}$ & $\begin{array}{l}\text { Computationally } \\
\text { complex for } \\
\text { complex } \\
\text { background images }\end{array}$ & $\begin{array}{l}\text { Best } \\
\text { preprocessing } \\
\text { required. }\end{array}$ \\
\hline
\end{tabular}


As there is no single method sufficient for all types of images for localization, one can use the text extraction techniques for localization or detection of license plate characters because the characters of the vehicle number plates are almost unique in size( for standard vehicle number plates) while considering other text around the plate region as noise. Noise removal techniques can be used as post processing for effective localization of the vehicle number plates. Advanced algorithms for text extraction should be developed for extracting text of only number plate region.

\section{REFERENCES}

[1] Zhang, Jia, Xiangiian He, "A Fast Algorithm for License Plate Detection in Various Conditions," 2006.IEEE International Conference on Systems, Man, and Cybernetics October 8-11, 2006.

[2] Cousineau, Matt, "The Global War on Terror and Automatic License Plate Recognition," Canadian Review of Sociology, Vol. 50, No.1, February 2013.

[3] Amninder kaur, Sonika jindal, Richa jindal, "License Plate Recognition using Support Vector Machine (SVM)", International Journal of Advanced Research in Computer Science and Software Engineering Vol. 2, issue 7, July 2012.

[4] Xin Li, "Vehicle License Plate Detection and Recognition," MS Thesis, December 2010.

[5] Shan Du, Mahmoud Ibrahim, Mohamed Shehata, "Automatic License Plate Recognition (ALPR): A State of the Art Review," IEEE Transactions On Circuits And Systems For Video Technology, 2011.

[6] S.Wang and H.Lee, "Detection and recognition of license plate characters with different appearances," Int. Conf.Intell. Transp. Syst., vol. 2, pp. 979-984, 2003.

[7] Satadal Saha , Subhadip Basu and Mita Nasipuri, "Automatic Localization and Recognition of License Plate Characters for Indian Vehicles," Int. J Comp Sci. Emerging Tech Vol-2 No 4 August, 2011.

[8] Dr. P.K.Ssuri, Dr. Ekta Walia, "Vehicle Number Plate Detection using Sobel Edge Detection Technique," IJCST Vol. 1, Issue 2, December 2010.

[9] PeterTarabek, "A Real-Time License Plate Localization Method Based on Vertical Edge Analysis," Proceedings of the Federated Conference on Computer Science and Information Systems pp. 149-154, 2012.

[10] Dhawal Wazalwar, Erdal Oruklu, Jafar Saniie,“A Design Flow for Robust License Plate Localization and Recognition in Complex Scenes,"Journal of Transportation Technologies, 2012, 2, 13-21.
[11] Humayun K.Sulehria, Ye Zhang, DanishIrfan, "Mathematical Morphology Methodology for Extraction of Vehicle Number Plates" International Journal Of Computers, Issue 3, Volume 1, 2007.

[12] Phalgun Pandya, Mandeep Singh, "Morphology Based Approach To Recognize Number Plates in India" International Journal of Soft Computing and Engineering (IJSCE),Volume-1, Issue-3, July 2011.

[13] Kaushik Deb, Hyun-Uk Chae, "Vehicle License Plate Detection Method Based on Sliding Concentric Windows and Histogram, "Journal Of Computers, Vol. 4, No. 8, August 2009.

[14]Haiqi Huang, Ming Gu, "An Efficient Method of License Plate Location in Natural-scene Image," Fifth International Conference on Fuzzy Systems and Knowledge Discovery, 2008IEEE.

[15] C.N.Anagnostopoulos, I.Giannoukos, A.Psyllos, V.Loumos and E.Kayafas, "IntegratedVehicle Recognition and Inspection System to ImproveSecurity in Restricted Access Areas," $201013^{\text {th }}$ International IEEE Annual Conference on Intelligent Transportation Systems Madeira Island, Portugal, September 19-22, 2010.

[16] Halina Kwaśnicka and Bartosz Wawrzyniak, "License plate localization and recognition in camera pictures,"AIMETH 2002 - Artificial Intelligence Methods November 13-15, 2002, Gliwice, Poland.

[17] R.C.Gonzalez, R.E.Woods, Digital Image Processing.2ded., Prentice Hall, Englewood Cliffs, NY, 2007

[18] Nadir Charniya, Automated Car License Plate Localization using Wavelet Analysis",International Journal of Computer Applications (0975 - 8887) Volume 42- No.2, March 2012.

[19] Shyang-Lih Chang, Li-Shien Chen, Yun-Chung Chung, "Automatic License Plate Recognition," IEEE Transactions On Intelligent Transportation Systems, Vol. 5, No. 1, March 2004.

[20] Lisheng Jin , Huacai Xian , Jing Bie , Yuqin Sun ,Haijing Hou, "License Plate Recognition Algorithm for Passenger Cars in Chinese Residential Areas," Sensors 2012.

[21] Joaqu'in Olivares, Jos'e M. Palomares, Jos'e M. Soto, and Juan Carlos G'amez, "License Plate Detection Based on Genetic Neural Networks, Morphology, and Active Contours,” Springer-Verlag Berlin Heidelberg 2010.

[22] B. Hongliang and L. Changping, "A hybrid license plate extraction method based on edge statistics and morphology," in Proc. ICPR, 2004,pp. 831-834. 